\title{
A hematoma confined to the center of the abdomen
}

\author{
MON-ING TSAI • CHIEN-CHENG CHEN • CHIH-HUANG LI • \\ TE-FA CHIU • JIH-CHANG CHEN • CHENG-HSIEN HSIEH
}

\author{
CHENG-HSIEN, HSIEH ( $\square$ ) \\ Department of Emergency Medicine \\ Chang Gung Memorial Hospital \\ No.5, Fusing St., Gueishan Township \\ Taoyuan County, 333, Taiwan, ROC \\ Tel: 886-3-3281200 ext. 2505 \\ Fax: 886-3-3287715 \\ E-mail: soga_hsieh@yahoo.com.tw \\ MON-ING, TSAI • \\ CHIEN-CHENG CHEN • \\ $\mathrm{CHIH}-\mathrm{HUANG} \mathrm{LI} \cdot$ \\ TE-FA CHIU • \\ JIH-CHANG CHEN • \\ CHENG-HSIEN, HSIEH \\ Affiliation \\ Department of Emergency Medicine \\ Chang Gung Memorial Hospital \\ Keelung, Taiwan1 \\ Department of Emergency Medicine \\ Chang Gung Memorial Hospital \\ Linko, Taiwan2 \\ Department of Radiology \\ Chang Gung Memorial Hospital \\ Linko, Taiwan3 \\ College of Medicine \\ Chang Gung University \\ Tao Yuan, Taiwan4
}

\begin{abstract}
Spontaneous rupture of a hepatocellular carcinoma is a rare and lethal complication in the emergency department. A caudate lobe hepatoma rupture is even rarer. It can be treated with vascular embolization, surgical intervention or supportive care. A 70-year-old woman with underlying hepatocellular carcinoma presented to our emergency department with severe abdominal pain encompassing the entire region for half a day. Abdominal computer tomography scans with and without contrast medium revealed a large hematoma confined to the lesser sac of the abdomen. It was initially diagnosed as a ruptured aneurysm. A ruptured caudate lobe hepatoma with acute hemorrhage into the lesser sac was diagnosed after reviewing and discussing the imaging findings with the radiologist. The patient was treated with supportive care without vascular embolization or surgical intervention because there was no imaging evidence of active contrast extravasation and the vital signs were within the normal range. After reviewing the literature, our case appears to be the second only case treated with supportive care and discharged without complications.
\end{abstract}

Keywords: hepatoma, hepatocellular carcinoma, caudate, rupture, peritonitis

\section{Introduction}

Spontaneous rupture of a hepatocellular carcinoma can cause massive blood loss and immediate hemodynamic change. Caudate lobe hepatoma rupture is rare and it may present as an exophytic hematoma in the peritoneum. Only 8 cases could be found in the literature. Treatments include vascular embolization, surgical intervention and supportive care.

\section{Case report}

A 70-year-old woman presented to our emergency department with severe abdominal pain encompassing the entire region, which had persisted for half a day. She had underlying hepatitis- $C$ for more than 20 years, and hepatitis- $C$ related liver cirrhosis (Child $A$ ) for about 3 years. About 1 year prior, routine ultrasonography revealed small nodules in the liver that were subsequently confirmed to be a hepatoma by fine needle aspiration. The abdominal pain was acute in onset, 
persistent, and not relieved by painkillers or fasting. The pain also worsened with coughing or walking and was without migration or radiation. Her initial temperature was $36.6^{\circ} \mathrm{C}$, heart rate was 77 beats per minute, respiratory rate was 17 breaths per minute, and blood pressure was 124/66 mmHg. Physical examination disclosed diffuse abdominal tenderness with rebounding pain. Initial chest X-ray and left decubitus abdominal $X$-ray showed no free air. Laboratory evaluation demonstrated a white blood cell count of $13600 / \mathrm{mm}^{3}$ with $66 \%$ segmented neutrophils, a hemoglobin of $9.8 \mathrm{~g} / \mathrm{dl}$, platelet count of $183,000 /$ $\mathrm{mm}^{3}$, lipase levels of $53 \mathrm{U} / \mathrm{L}$ (normal range $<55$ ), prothrombin time of 11.2 seconds (normal plasma mean of 10.7 seconds), activated partial thromboplastin time of 26.2 seconds (normal plasma mean of 25.1 seconds) and $C$ reactive protein levels of $13.27 \mathrm{mg} / \mathrm{L}$ (normal range $<5$ ). Other laboratory results were all within normal limits. Abdominal computer tomography scans with and without contrast revealed a large hematoma confined to the lesser sac of the abdomen (figure 1 and 2). We initially thought it may have been a ruptured aneurysm, but a ruptured caudate lobe hepatoma with acute hemorrhage into the lesser sac was diagnosed after reviewing and discussing the imaging findings with the radiologist (figure 3 and 4 ). The patient was treated with supportive care without vascular embolization or surgical intervention because there was no imaging evidence of active contrast extravasation and the vital signs were within the normal range.

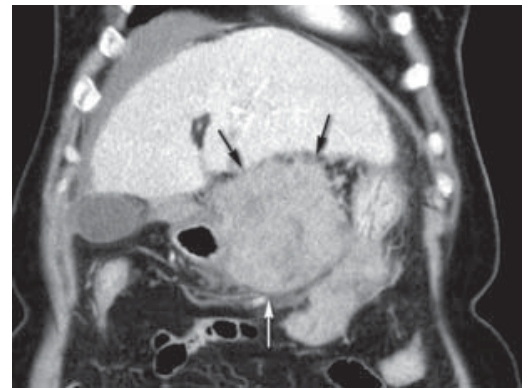

Figure 1. Contrast CT image showing a large hematoma in the lesser sac.

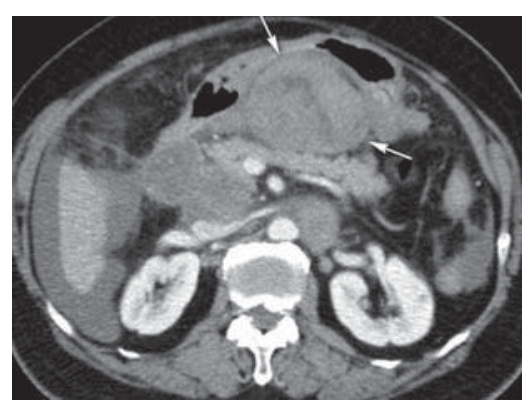

Figure 2. Coronal reformatted CT image showing an exophytic hematoma below the liver, left and lateral to the duodenum.

She stayed in our hospital for 7 days and was discharged after transarterial embolization of the residual hepatoma. Follow- up 30 days and 90 days later revealed no complications.

\section{Discussion}

Tumor rupture is a serious and lifethreatening complication of hepatoma. Ruptured tumors may cause massive bleeding into the peritoneum and significant hemodynamic change. Treatment includes vascular embolization, surgical intervention, and supportive care. The caudate lobe is a rare location for hepatoma rupture and may result

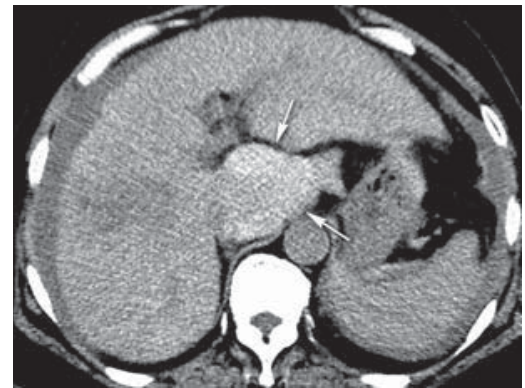

Figure 3. Pre-contrast CT image showing hyperdensity (arrows) surrounding the caudate lobe in the lesser sac corresponding to acute hemorrhage.

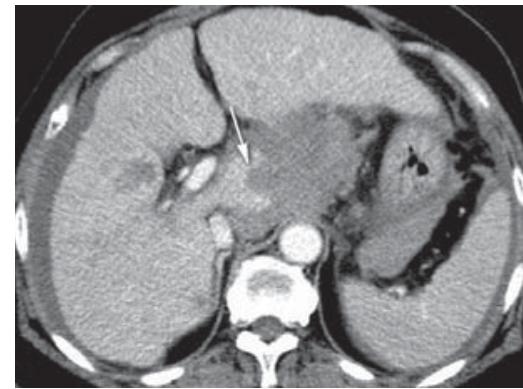

Figure 4. Focal bulging of the caudate lobe with a parenchymal defect on the medial aspect was a ruptured hepatoma (arrow).

in exophytic hematoma into the peritoneum. Only eight previous cases were found in the literature. (1-4) Four cases were treated with vascular embolization, $(1,3)$ one case was treated with immediate surgery after vascular embolization failed, (4) one case was sent to surgery after abdominal CT confirmed the diagnosis, (2) one case was in hypovolemic shock and died 1 day later without any treatment, (3) and one case received supportive care and survived for another 104 days. (3) Our case may be the second only case treated with supportive care and discharged without complications.

\section{REFERENCES}

1. Takahashi A, Saito H, Takahashi Y, Sato A, Takagi T, Irisawa A, et al. Cyst-like extension of hepatic subcapsular bleeding caused by ruptured hepatocellular carcinoma into the bursa omentalis. Hepatol Res 2009;39:1010-4.

2. Lin CH, Hsieh HF, Chou SJ, Yu JC, Chen TW, Hsieh CB. Ruptured caudate lobe hepatocellular carcinoma presents with lesser sac tumor. Rev Esp Enferm Dig 2006;98:703-4.

3. Iwasaki Y, Tani I, Nakajima Y, Ishikawa T, Umeda S, Kusano S. Lesser sac hematoma as a sign of rupture of hepatocellular carcinoma in the caudate lobe. Eur Radiol 2001;11:422-6.

4. Nakao A, Matsuda T, Koguchi K, Funabiki S, Mori T, Kobashi K, et al. Spontaneous rupture of hepatocellular carcinoma of the caudate lobe. Anticancer Res 2000;20:2223-7. 